\title{
A periodic planning model for maritime transportation of crude oil
}

\author{
Atiq Siddiqui $\cdot$ Manish Verma $\cdot$ David Tulett
}

Received: 4 May 2013/Accepted: 14 August 2013/Published online: 29 August 2013

(C) Springer-Verlag Berlin Heidelberg and EURO - The Association of European Operational Research Societies 2013

\begin{abstract}
Crude oil is primarily transported through sea using very large tankers. Efficient scheduling of these tankers is challenging as well as critical given long lead times, tight delivery time windows and high operational costs. We attempt to solve such a scheduling problem for an oil supplier facing supply quota and port capacity constraints. A mixed-integer programming formulation and two timedependent solution techniques are proposed. Numerical results suggest that computing time was a function of the number, starting location, and time to availability of tankers at the supply sources. Finally, a time-based decomposition technique is presented to solve large problem instances, illustrating substantial reductions in computing time for marginally worse-off solutions.
\end{abstract}

Keywords Crude oil tankers - Marine transportation $\cdot$ Mixed-integer programming $\cdot$ Periodic planning $\cdot$ Scheduling

\section{Introduction}

Crude oil, one of the primary sources of energy, is procured from sources that are limited and dispersed around the world-thereby necessitating transportation over long distances. Maritime transportation, the primary mode for intercontinental

\footnotetext{
A. Siddiqui · D. Tulett

Faculty of Business Administration, Memorial University of Newfoundland, St. John's, Canada e-mail: atiq.siddiqui@mun.ca

D. Tulett

e-mail: dtulett@mun.ca

M. Verma ( $\square)$

DeGroote School of Business, McMaster University, Hamilton, Canada

e-mail: mverma@mcmaster.ca
} 
movement of crude oil, accounts for 1.7 billion tons annually (Rodrigue et al. 2009), the bulk of which is carried through the very large and ultra large crude carriers (i.e., VLCC and ULCC). These are the largest classes of oil tankers with carrying capacity exceeding $200 \mathrm{~K}$ tons and each costs tens of thousands of dollars a day, which translates into 10-22 USD/ton of oil shipped (Cheng and Duran 2004).

Such planning problems, involving use of tankers to meet a set of demands, are generally termed as oil tanker routing and scheduling problem in maritime transportation (Hennig et al. 2012). In this work, we focus on the periodic routing and scheduling problems faced by an oil supplier with customers around the globe. The supplier makes use of a fleet of heterogeneous vessels (mainly VLCC and ULCC tankers) to deliver a single grade of crude oil in response to multiple customer orders in one or more periods. Typically, customers release orders which in turn are determined by a number of internal and external factors (Kuo and Chang 2008; Luo and Rong 2009). Each order has to be delivered within a specified time window, but could be split amongst multiple shipments due to the size of an order. For example, demand for half a million tons of crude will be met using more than one tanker. Normally, for such suppliers the planning horizon would be 3 months, though it could be more (Brown et al. 1987). Finally, note that this supplier may constantly receive new and/or changes on earlier orders, which implies consequent updating and/or preparing new routing \& scheduling plans. We attempt to solve such a problem by proposing a periodic requirement scheduling (PRS) approach, which contains a mixed-integer programming formulation and two distinct time-dependent solution techniques.

The rest of the paper is organized as follows: "Literature review" outlines the current state of literature, and then distinguishes our current work. "Problem description" provides a brief description of the problem, followed by a discussion on the mathematical model and the solution methods in "Analytical framework". "Computational experiments" discusses the features of a realistic problem instance and the solution, and then outlines three special cases of the problem which are further analyzed for managerial insights. "Decomposition heuristic" makes use of illustrative examples to demonstrate the effectiveness of the proposed decomposition heuristic, while conclusions are contained in "Conclusion".

\section{Literature review}

Scheduling research in maritime transportation has attracted relatively less attention compared to other modes of transportation (Christiansen et al. 2004), which is often attributed to factors such as lack of structured planning and need for customized solutions. We invite the reader to refer to Ronen $(1983,1993)$ and Christiansen et al. $(2004,2007)$ for excellent discussions on these issues. Although problems involving maritime transportation of crude oil have been studied since the 1950s, the attention has been limited and intermittent. The relevant works can be categorized under two streams, i.e., with and without strict cargo specification. We elaborate on the two terms in the following paragraphs, but it is pertinent to note that the resulting approaches were fundamentally different. Next, we chronologically review the 
works under the first stream and then comment on the limitations, before outlining the basics of the second stream-which also forms the basis of our work.

In the first stream, early research works focused on the fuel oil transportation problem for the US Navy. For this problem, Dantzig and Fulkerson (1954) presented an integer programming model that minimized the total number of tankers under a fixed oil supply schedule. They considered a homogeneous fleet, full shipload paired demand (i.e., supply and demand location matching), and used linear programming approximation to solve the problem. Briskin (1966) and Bellmore (1968) extended the same problem, where the former modified the full shipload specification from a single port to a cluster of ports, while the latter studied the under capacity fleet situation. More recently, Brown et al. (1987) proposed an optimization model to solve a routing and scheduling problem faced by Chevron Shipping Company (CSC), a major oil company with homogeneous fleet. This study focused on crude oil shipment from the Middle East to Europe and North America, and endeavored to determine the schedule for a given set of cargo specified by quantities, ports (loading and discharging), and dates (loading and delivery). To ease complexity and to facilitate computation, the problem was modeled as an elastic set partitioning problem where a column generation technique was employed to generate a partial set of complete feasible schedules. The CSC problem was also investigated in two subsequent works. Perakis and Bremer (1992) proposed an integer programming formulation to schedule crude oil tankers, whereas Bremer and Perakis (1992) outlined the algorithmic details for a lab implementation of the same problem. Their system generated feasible schedules for each vessel and then made use of an integer program to determine the overall optimal schedule; it was subsequently tested on a realistic scheduling problem instance faced by CSC. Bausch et al. (1998) developed a decision support system, driven by a mathematical programming model, which could be used daily to schedule the dispatch of liquid bulk products by ships and barges amongst several local plants, bulk distribution terminals and industrial customers. In contrast, Sherali et al. (1999) proposed a mixed-integer programming approach to study the scheduling of a heterogeneous fleet of compartmentalized ships that could be used to transport a set of non-mixing cargo, i.e., comprised of crude oil and petroleum products. The resulting mathematical formulation was rather complex, and hence an alternate aggregate model was developed and solved using a specialized rolling horizon heuristic. In a recent work, Kobayashi and Kubo (2010) studied the oil transportation problem in a tramp setting, i.e., a shipping company contracted to deliver a set of cargoes. The mathematical program, involving local transportation of numerous petroleum products, was decomposed into two set partitioning problems of cargo pairing and tanker routing, which were then solved using a column generation technique. In another standalone work, Kobayashi (2010) addressed a similar problem in a more strategic setting, i.e., they considered long-term planning with multiple planning stages and proposed an approximate dynamic programming approach to solve the problem.

All of the above studies assume a given set of cargoes for which a delivery schedule has to be made. More specifically, they are based on strict cargo specifications including shipment sizes, paired pickup and delivery locations, and delivery time windows. This may not be the best approach for two reasons. First, given the nature of crude oil supply, several shipments are generally needed to fulfill a customer's requirement corresponding to a time period, and thus exactness of a 
particular delivery becomes less critical (Brown et al. 1987). Second, large buffer stocks are maintained at customer locations which further underscores the need to not strictly specify the size of a particular shipment. Thus, it is easy to contend that by matching the structure of demand to the available resources, one can not only ensure better utilization of assets (i.e., tankers) but also generate more efficient schedules. As indicated earlier, the demand for crude oil is periodically assessed by customers (i.e., refineries), and then a requirement plan spanning typically up to 3 months is laid out (Brown et al. 1987).

The second stream of research includes those works wherein the cargo set is not strictly specified. McKay and Hartley (1974) were the first who argued for this approach, and presented an integer programming formulation for oil tanker scheduling that permitted arbitrary splitting of ordered quantities. Their model, developed for the US Defense, aimed at minimizing the cost of operations and fuel purchase at loading ports. This approach did not receive further attention until recently. Hennig et al. (2012) proposed a framework to solve maritime transportation of multiple grades of crude oil in the presence of both pickup and delivery time windows, and unpaired supply and demand quantities. The proposed model permits arbitrary splitting of both loading and unloading quantities amongst multiple shipments, and also multiple pickups and deliveries in a single voyage. The model selects the optimum route from a set of pre-generated tanker routes, and was tested on a number of random problem instances. In an effort to position their work with respect to the general transportation and vehicle routing literature, the authors have suggested that it be considered as split pickup and split delivery problem with time windows (i.e., SPSDWTW).

Though our work adheres to the basic premise of the second stream, it is fundamentally distinct from the existing literature in that it aims to provide sufficient capacities rather than specifying quantities to individual tankers when scheduling tanker trips. Note that this is a more robust approach since it allows a manager to postpone exact quantity assignment to a tanker until its arrival at a supply port, which in turn facilitates better management of small quantity adjustments and increased vessel utilization without changing tanker slates. Furthermore, in contrast to Hennig et al. (2012) who solve the problem for a transporter, we assume a more vertically integrated structure in that the transport company is owned by the oil supplier-and hence there are no quantity and time window restrictions at the supply sources. The latter points are important since they enabled us to incorporate more realistic upstream supply chain features such as supply points quota constraints, thereby facilitating more efficient logistics planning-which is consistent with the argument in favor of a more integrated approach in planning oil transportation (Chajakis 2000). Our modeling approach is also significantly different in the following additional ways: first, given the geographical clustering of both supply and demand locations typical to the problem, we allow periodic demand of a number of discharging locations-in close proximity - to be consolidated into a single demand thereby permitting solving larger realistic problem instances. Second, our mixed-integer programming formulation does not involve pre-generation of routes as in Hennig et al. (2012) since such an approach is problematic for large problem sizes. For example, we are assuming up to 30 tankers making multiple trips from multiple sources to multiple 
destinations, which could result in a very large number of possible routes. Third, since suppliers receive new and updated orders continually, while their resources are tied up as a result of current assignments, we also develop two time-dependent periodic planning approaches that capture the rolling horizon nature of the problem thereby facilitating updates to existing and/or development of new plans. Finally, since our optimization model allows splitting the loading quantities at the supply sources, which are then delivered fully to a single demand location within the specified time window, it could be viewed as a split pickup and delivery problem with time window (i.e., SPDWTW).

\section{Problem description}

In this section, we briefly discuss the managerial problem of interest and then outline the basic modeling assumptions.

At a higher level, the managerial problem involves determining efficient sailing schedules of a set of tankers being used to meet customer demands in a planning horizon. Furthermore, each customer demand is specified by a quantity and a specific delivery period (or time window). Note that a number of delivery periods constitute a planning horizon, which starts when orders are received and ends when all demand time windows are covered. Such problems are realistic and faced by most crude oil companies, such as Chevron and Kuwait Petroleum Corporation, which make use of a heterogeneous fleet of tankers on an existing network of routes between their supply and demand locations. The objective is to minimize the total cost of operations while meeting demand and adhering to various restrictions.

To make this more explicit, assume a number of customer locations, indexed by $d$, with different periodic demands. For example, $Q_{d_{i}}$ refers to the quantity demanded at location $d$ in period $i$, which has to be serviced by the supplier through its available supply sources or ports $s_{i}$ (Fig. 1). Typical requirement period (or time window) length for each of the orders could be a week, as dictated by the scope of the problem, while the corresponding planning horizon could be in months. Note that in Fig. 1 there are two demand locations and that each has two requirement periods within the given planning horizon. The crude oil supplier receives the respective demand information at a time denoted as $\pi$, and then needs to develop a schedule for its fleet such that specified cargo deliveries could be made within respective requirement periods. It is important to note that not all tankers are available at the supply ports right at the beginning of the planning horizon, since some may be en route to/from demand locations (i.e., anywhere in the network). For our problem instance, we assume that sufficient fleet is available at the beginning of the planning horizon.

It should be clear that this is a rather complicated problem, since the time until these tankers become available for loading depends on their locations in the network at the end of the previous schedule. We refer to those locations as artificial origins, which are indicated by nodes in Fig. 1 . For example, $t_{1}^{a}$ is the time when the first vessel becomes available for usage at the artificial origin indicated by the first demand point $d=1$. The decisions surrounding tanker scheduling are further 


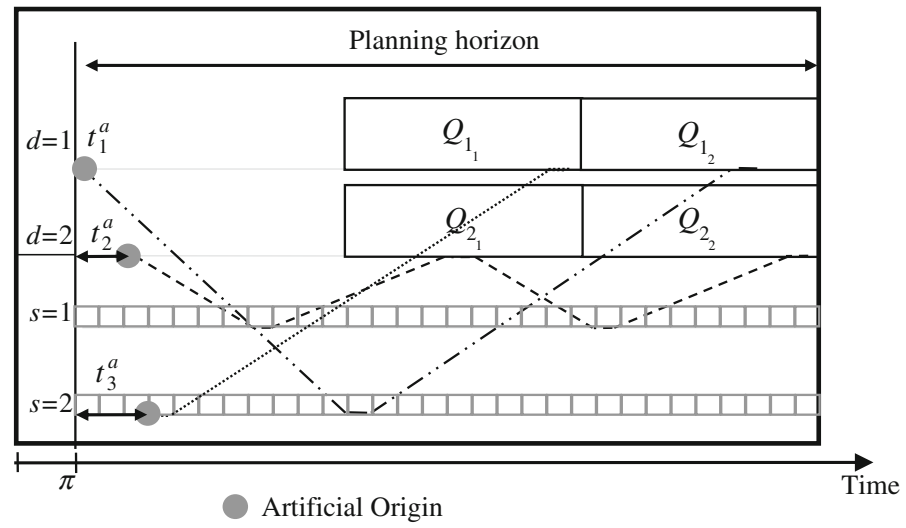

Fig. 1 Crude oil periodic requirement

complicated because of the capacity considerations at the supply ports on any given day (represented by small gray boxes) and the associated port or area-wise supplier quota restrictions (i.e., restriction on the amount of oil supplied through a port in a given period). In an effort to address such a problem, we propose a planning approach that considers the scheduling of tankers for periodic crude oil requirements, develops two time-dependent rolling horizon solution schemes, and exploits the natural demand structure and resource characteristics.

Before outlining the mathematical program in the next section, we list the six assumptions pertinent to the managerial problem outlined above: first, demand requirements (assuming a single grade of oil) for a specific planning horizon are known before the start of the respective planning horizon; second, all relevant costs to operate a tanker such as fuel and idling are known; third, every tanker picks up its cargo from a single supply source and delivers the entire shipment to a single demand location; fourth, no return cargoes are allowed (since crude carriers do not carry petroleum products); fifth, a heterogeneous fleet of owned and long-term time chartered VLCC/ULCC tankers is assumed, which go either to the ports capable of receiving them or to the lightering zones (unloading zones, to smaller vessels, in open sea); and sixth, tankers are allowed to start anywhere in the network (i.e., can assume the so-called artificial origins).

\section{Analytical framework}

In this section, we first outline the periodic requirements model and then discuss the time-dependent periodic planning solution methodologies, which together constitute the 'periodic requirement scheduling approach' or the PRS Approach.

\section{Periodic requirements model}

Before outlining the mathematical program, we define two terms that are integral to the formulation: First, a trip comprises of a loaded leg and an empty return leg. It 
should be evident that a tanker can make a number of trips during a planning horizon, and hence we introduce an index $j$ to keep track. In addition, we introduce $\tau_{v}$ to denote the maximum number of trips for any tanker $v$, which in turn is a function of the length of the planning horizon, and the quickest (shortest) trip tanker $v$ can make between a pair of supply-demand ports. We provide estimation details for this in "Solution and discussion". Second, a partial trip can indicate either a loaded leg or an empty return leg. This is important, since we assume the last utilized trip of a tanker to any requirement period be only a loaded leg, while to use the tanker for a subsequent scheduling plan, we assume the corresponding demand point to be the location where the tanker becomes available for the next requirement period (i.e., artificial origin). Note that, if an empty return leg is the last trip in a specific requirement period, then the tanker is available at the supply point in the following period. Finally, we observe that all the time-related parameters are continuous and determined based on average speed.

\section{Sets and indices}

$V \quad$ Set of available tankers or vessels, indexed by $v$

$S \quad$ Set of available supply points, indexed by $s$

$D \quad$ Set of demand points, indexed by $d$

$A \quad$ Set of artificial origins, indexed by $a$

$K \quad$ Number of periods on the supply side, indexed by $k$ (equal to total days in a planning horizon $P$ )

I Number of requirement periods at a customer location, indexed by $i$

$j$

Trip number index

Variables:

$$
\begin{aligned}
& x_{v s d}^{j}=\left\{\begin{array}{cc}
1 & \text { if vessel } v, \\
y_{v d s}^{j} & =\left\{\begin{array}{cc}
1 & \text { if vessel } v, \text { on a return-leg of trip } j, \text { travels from } d \text { to } s \\
0 \text { otherwise }
\end{array}\right. \\
0 \text { otherwise }
\end{array}\right.
\end{aligned}
$$

$w_{v d_{i}}^{j}=\left\{\begin{array}{c}1 \text { if vessel } v, \text { on trip } j, \text { delivers to a customer location } d \text { in period } i \\ 0 \text { otherwise }\end{array}\right.$

$$
\begin{aligned}
& y_{\text {vas }}^{0}=\left\{\begin{array}{c}
1 \quad \text { if vessel } v, \text { during trip } 0, \text { travels from } a \text { to } s \\
0 \text { otherwise }
\end{array}\right. \\
& z_{v s_{k}}^{j}=\left\{\begin{array}{c}
1 \text { if vessel } v, \text { starts its loaded leg at } s, \text { on } k^{\text {th }} \text { day } \\
0 \text { otherwise }
\end{array}\right.
\end{aligned}
$$

$b_{v}^{j}$ Waiting/Idling time of vessel $v$ (at supply point) before starting trip $j$.

$e_{v}^{j}$ Time until vessel $v$ starts loaded leg on trip $j$

$f_{v}^{j}$ Time until vessel $v$ finishes loaded leg on trip $j$ 


\section{Parameters}

$Q_{d_{i}} \quad$ Quantity of crude demanded at a customer location $d$ in week $i$

$C_{v} \quad$ The cargo carrying capacity of vessel $v$

$P \quad$ Planning horizon in days

$\mathrm{ALW}_{d} \quad$ Percentage allowance on periodic requirements at $d^{1}$

$C(s)_{k} \quad$ Available port capacity (in tons/day) at $s$ on the $k^{\text {th }}$ day of the planning horizon

$\gamma_{s} \quad$ Percentage distribution quota amongst supply ports ${ }^{2}$

$c_{v s d} \quad$ Cost to move vessel $v$, on a loaded leg from a supply point $s$ to a demand point $d$

$c_{v d s} \quad$ Cost to move vessel $v$, on a return leg from a demand point $d$ to a supply point $s$

$c_{\text {vas }} \quad$ Cost to move vessel $v$, from its artificial origin $a$ to a supply point $s$

$I C_{v} \quad$ Idling cost per unit time of vessel $v$

$t_{v s d} \quad$ Time needed by vessel $v$ to travel from $s$ to $d$

$t_{v d s} \quad$ Time needed by vessel $v$ to travel from $d$ to $s$

$t_{\text {vas }} \quad$ Time needed by vessel $v$ to travel from $a$ to $s$

$t_{v}^{a} \quad$ Time until a vessel is available for service at its artificial origin

$l_{v} \quad$ Time needed to load vessel $v$

$u_{v} \quad$ Time needed to unload vessel $v$

$t(E)_{d_{i}} \quad$ Earliest delivery time at $d$ in period $i$

$t(L)_{d_{i}} \quad$ Latest delivery time at $d$ in period $i$

$\tau \quad$ Maximum number of allowable trips in a planning horizon

(PBM)

$$
\begin{aligned}
& \min \sum_{v \in V} \sum_{1 \leq j \leq J} \sum_{s \in S} \sum_{d \in D} c_{v s d} x_{v s d}^{j}+\sum_{v \in V} \sum_{1 \leq j \leq J} \sum_{d \in D} \sum_{s \in S} c_{v d s} y_{v d s}^{j}+\sum_{v \in V} \sum_{a \in A} \sum_{s \in S} c_{v a s} y_{v a s}^{0} \\
& \quad+\sum_{v \in V} \operatorname{IC}_{v}\left(\sum_{1 \leq j \leq J} b_{v}^{j}\right)
\end{aligned}
$$

Subject to:

Demand fulfillment:

$$
\sum_{v \in V} \sum_{1 \leq j \leq J} C_{v} w_{v d_{i}}^{j} \geq Q_{d_{i}}\left(1-\mathrm{ALW}_{d}\right) \quad \forall d \in D, i \in I
$$

Delivery window:

\footnotetext{
1 This is to allow contractual flexibility on actual order or periodic requirements by a customer, which is an acceptable practice in the crude oil industry. This is captured through constraint set (2) in the optimization model, and further elaborated in the paragraphs following the model.

2 This depicts periodic supply quotas imposed on any specific supply port due to, for instance, policy or upstream network restrictions. We elaborate more on this in "Periodic requirements model".
} 


$$
\begin{gathered}
\text { Earliest delivery }: f_{v}^{j}+P \sum_{s \in S} x_{v s d}^{j}-t(E)_{d_{i}} w_{v d_{i}}^{j}-P w_{v d_{i}}^{j} \geq 0 \\
\forall v \in V, 1 \leq j \leq \tau, d \in D, i \in I \\
\text { Latest delivery : } f_{v}^{j}-P \sum_{s \in S} x_{v s d}^{j}-P-t(L)_{d_{i}} w_{v d_{i}}^{j}+2 P w_{v d_{i}}^{j} \leq 0 \\
\forall v \in V, 1 \leq j \leq \tau, d \in D, i \in I \\
f_{v}^{1}=t_{v}^{a}+\sum_{a \in A} \sum_{s \in S} t_{v a s} y_{v a s}^{0}+b_{v}^{1}+\sum_{s \in S} \sum_{d \in D}\left(l_{v}+t_{v s d}+u_{v}\right) x_{v s d}^{1} \quad \forall v \in V \\
f_{v}^{j}=f_{v}^{j-1}+\sum_{d \in D} \sum_{s \in S} t_{v d s} y_{v d s}^{j-1}+b_{v}^{j}+\sum_{d \in D} \sum_{s \in S}\left(l_{v}+t_{v s d}+u_{v}\right) x_{v s d}^{j} \\
\forall v \in V, 2 \leq j \leq \tau
\end{gathered}
$$

Structural:

$$
\begin{gathered}
\sum_{d \in D} \sum_{1 \leq i \leq I} w_{v d_{i}}^{j} \leq 1 \quad \forall v \in V, \quad 1 \leq j \leq \tau \\
\sum_{d \in D} x_{v s d}^{1} \leq \sum_{a \in A} y_{v a s}^{0} \quad \forall v \in V, s \in S \\
\sum_{a \in A} \sum_{s \in S} y_{v a s}^{0}=1 \quad \forall v \in V \\
\sum_{d \in D} x_{v s d}^{j} \leq \sum_{d \in D} y_{v d s}^{j-1} \quad \forall v \in V, 2 \leq j \leq \tau, s \in S \\
\sum_{s \in S} y_{v d s}^{j} \leq \sum_{s \in S} x_{v s d}^{j} \quad \forall v \in V, d \in D \\
\sum_{s \in S} \sum_{d \in D} x_{v s d}^{j} \leq 1 \quad \forall v \in V, \quad 1 \leq j \leq \tau \\
\sum_{d \in D} \sum_{s \in S} y_{v d s}^{j} \leq 1 \quad \forall v \in V, \quad 1 \leq j \leq \tau \\
\sum_{s \in S} \sum_{d \in D} x_{v s d}^{j} \leq \sum_{s \in S} \sum_{d \in D} x_{v s d}^{j-1} \quad \forall v \in V, \quad 2 \leq j \leq \tau
\end{gathered}
$$

Supply:

A. (Port capacity):

$$
\begin{gathered}
\sum_{d \in D} x_{v s d}^{j}-\sum_{1 \leq k \leq K} z_{v s_{k}}^{j}=0 \quad \forall v \in V, s \in S, 1 \leq j \leq \tau \\
\sum_{v \in V} C_{v} z_{v s_{k}}^{j} \leq C(s)_{k} \quad \forall s \in S, k \in K
\end{gathered}
$$




$$
\begin{gathered}
\text { Earliest arrival: } e_{v}^{j}-(k-1) z_{v s_{k}}^{j}+P\left(\sum_{d \in D} x_{v s d}^{j}-z_{v s_{k}}^{j}\right) \geq 0 \\
\forall v \in V, 1 \leq j \leq \tau, s \in S, k \in K \\
\text { Latest arrival: } e_{v}^{j}-z_{v s_{k}}^{j}(k-2 P)-P\left(\sum_{d \in D} x_{v s d}^{j}+1\right) \leq 0 \\
\forall v \in V, 1 \leq j \leq \tau, s \in S, k \in K \\
e_{v}^{1}=t_{v}^{a}+\sum_{a \in A} \sum_{s \in S} t_{v a s} y_{v a s}^{0}+b_{v}^{1} \quad \forall v \in V \\
e_{v}^{j}=f_{v}^{j-1}+\sum_{d \in D} \sum_{s \in S} t_{v d s} y_{v d s}^{j-1}+b_{v}^{j} \quad \forall v \in V, 2 \leq j \leq \tau
\end{gathered}
$$

B. (Supply distribution quota):

$$
\sum_{v \in V} \sum_{1 \leq j \leq J} \sum_{d \in D} C_{v} x_{v s d}^{j} \leq \gamma_{s} \sum_{v \in V} \sum_{1 \leq j \leq J} \sum_{s \in S} \sum_{d \in D} C_{v} x_{v s d}^{j} \quad \forall s \in S
$$

Variable types:

$$
\begin{aligned}
& x_{v s d}^{j} \in\{0,1\}, \quad y_{v d s}^{j} \in\{0,1\}, \quad w_{v d_{i}}^{j} \in\{0,1\}, \quad z_{v s_{k}}^{j} \in\{0,1\} \\
& b_{v}^{j} \geq 0, \quad f_{v}^{j} \geq 0, \quad e_{v}^{j} \geq 0
\end{aligned}
$$

$(\mathrm{PBM})$ is a mixed-integer programming formulation, where the objective function represents the total cost of operations resulting from all the trips made by crude oil tankers over a planning horizon. Note that (1) includes the cost of all loaded- and return legs, the cost for traveling from artificial origins to supply points, and the cost of idling at supply points. It may be evident that given the capital intensive nature of operation and the goal of matching demand structure to fleet characteristics, one would expect most of the oil tankers to travel full and some almost full, which could result in some difference between estimated and actual costs.

For expositional reasons, constraints (2)-(20) are divided into four categories: demand fulfillment; delivery window; structural; supply. Constraints (2) ensure that the total committed delivery capacity to location $d$ in period $i$ equals or exceeds the requirement. A common practice in crude oil supply contracts is to allow a range within which actual quantity can be delivered (Sherali et al. 1999). The specified percentage allowance, $\mathrm{ALW}_{d}$, in fact facilitates better utilization of tanker capacities since the actual delivery amount need not be exactly equal to the periodic requirement. It is important to note that the proposed model will deliver a set of vessels that could be used by the transport manager to meet demand during a requirement period, but the manager has to distribute the ordered quantity amongst the recommended vessels.

Constraints (3)-(5) concern delivery time windows and associated variables. Constraints (3) ensure that vessel $v$ on trip $j$ can make delivery at demand location $d$ in period $i$, if and only if, the vessel visits the specific customer on that trip (i.e. $x_{v s d}^{j}=1$ ), and does it within the allowed time window, i.e., $w_{v d_{i}}^{j}=1$ is set to 1 , 
only if $x_{v s d}^{j}=1$ and that $f_{v}^{j} \in\left[t(E)_{d_{i}}, t(L)_{d_{i}}\right]$. Constraints (4) estimate the time until vessel $v$ is available at demand point $d$ during the first trip, whereas constraints (5) indicate vessel availability for all other used trips. Please note that we have defined $\tau$ as the maximum allowable trips during a planning horizon, which bounds the actual number of used trips. Assuming $\bar{j}$ to be the last used trip by vessel $v$, then for the remaining unused trips, (5) yield $f_{v}^{\bar{j}+o}=f_{v}^{\bar{j}} \forall 1 \leq o \leq(\tau-\bar{j})$.

Constraints (6)-(13) are the structural integrity of the problem. Constraints (6) ensure that vessel $v$ on trip $j$ makes a single delivery of the entire cargo, while (7) ensures that vessel $v$ has to arrive at $s$ before it can leave for $d$ on the very first trip. Constraints (8) say that vessels with an artificial origin at a demand point at the beginning of a planning horizon, and not scheduled to make a delivery in the current plan, should return to a supply port. This is important since it would be unrealistic to keep such vessels stationed at a demand point. It is also pertinent to note that since it is unrealistic to let the tankers wait at different locations when not in use, it is necessary to use the concepts of "artificial origin" and "partial trips". To that end, constraints (9) ensure that vessels leaving $s$ during loaded leg of trip $j(\geq 2)$ can do so only if these vessels had return leg to $s$ during trip $j-1$. Similarly, constraints (10) ensure that a vessel during a trip $j$ leaves on return leg from the same demand point $d$, that it had reached during this trip. Constraints (11) and (12) ensure that at most one loaded leg and one return leg are assigned per trip, respectively. For unused trips $x_{v s d}^{j}$ and $y_{v d s}^{j}$ are set to zero. Finally, constraints (13) ensure that trip $j$ for a vessel is utilized only if $j-1$ has already been utilized.

Constraints (14)-(18) enforce port capacity (in tons/day). Constraints (14) make sure that $z_{v s_{k}}^{j}$ is set to 1 , when a delivery is undertaken by a vessel $v$ from a supply point $s$ during trip $j$ (i.e., for some $x_{v s d}^{j}=1$ ), and the corresponding assignment in terms of available port capacity is accounted for in constraints (15). To identify the $k^{\text {th }}$ day a vessel makes itself available at the supplier port, i.e., corresponding to $z_{v s_{k}}^{j}=1$, and that a delivery is consequently made, i.e., $x_{v s d}^{j}=1$ during trip $j$, constraints (16) is used. The time until that loaded leg can start $\left(e_{v}^{j}\right)$ is determined using constraints (17) and (18). In an effort to capture quantity distribution amongst competing supply points within a jurisdiction, we introduce constraints (19). This could be mandated because of political or economic or upstream supply network restrictions. Finally, constraints (20) depict the sign restriction constraints.

\section{Solution methodology}

Although schedules are generated for a specific time horizon, crude oil transportation problem has a rolling horizon nature, and hence scheduling for any particular planning horizon cannot be done in isolation since it will depend on the events of the preceding planning period. In addition, order adjustments based on new information often results in alterations in the current cargo slate of a vessel. In an effort to capture these two attributes, we propose two time-dependent periodic planning (TDP) schemes to solve (PBM) for the two given situations. Both schemes make use of a deterministic rolling horizon setting, wherein a decision maker makes 
use of the new information to plan and/or update delivery operations. In other words, the planning horizon is rolled over again and again as new information becomes available. Recent examples of this approach include Al-Khayyal and Hwang (2007), who developed an inventory routing and scheduling model in multicommodity bulk shipping, and Rakke et al. (2011), who developed a rolling horizon solution methodology for a liquefied natural gas inventory routing problem.

We first define each schedule and the start time in a sequence of time-dependent schedules, such that Schedule $_{n}$ will start at $\pi_{n}$ and depends on Schedule Sh-1 $_{n}$ which starts at $\pi_{n-1}$. Note that $\pi_{n-1}<\pi_{n}$, otherwise two schedules can be merged and solved as a single problem. For example, in Fig. 2, Schedule precedes Schedule $_{2}$, and the artificial origins for the three vessels in the latter schedule are their terminating positions in the former schedule. The dashed line scheme indicates the movement of the three vessels, supply and demand locations are represented by $s$ and $d$, respectively, and the element of time extends across the two schedules. Next, we outline the two solution schemes.

\section{TDP1: no schedule change allowed}

This scheme is intended to solve the problem instance depicted in Fig. 2, where the availability of new periodic requirements during the current schedule does not impact the rest of the schedule, and the ship availabilities for the new schedule are initialized based on the final vessel availabilities posed by the current schedule. Such a situation arises when any flexibility or adjustment in a schedule is not allowed. For expositional reasons, we provide a brief outline of the proposed algorithm, and invite the reader to consult "Appendix A" for complete details.

The algorithm starts by assigning an index $n$ to a new scheduling plan, i.e., Schedule $_{n}$, and if $n=1$ (i.e., planning done for the first time), it calls for initializing all the parameters, generating and solving the model. For the following plans $(n>1)$, the algorithm first defines the set of vessels used in the current schedule that would be carried to the next schedule (excluding any vessel leaving the fleet). Subsequently, an empty set of artificial origins is defined and assigned to the corresponding ship locations (i.e., supply points for unused ships and the last-visited demand locations for the used ships), followed by the time when each vessel would become available for service (viz. zero for unused ships and finish time of the last loaded legs for the used ships). Any new vessel, with respective artificial origin and time to availability parameters, is added to the plan-which is solved in the last step.

\section{TDP2: schedule change permitted}

Unlike TDP1, in TDP2 availability of new periodic requirements and/or order adjustments do have an impact on the current schedule. As demand information arrives during the current Schedule $_{n-1}$, its unfulfilled part is appended and/or modified with the new Schedule ${ }_{n}$. This implies attaching the unfulfilled part of the current schedule with the requirements of the new schedule, and then solving the resulting new problem. The scheme allows the new problem to be initialized based 


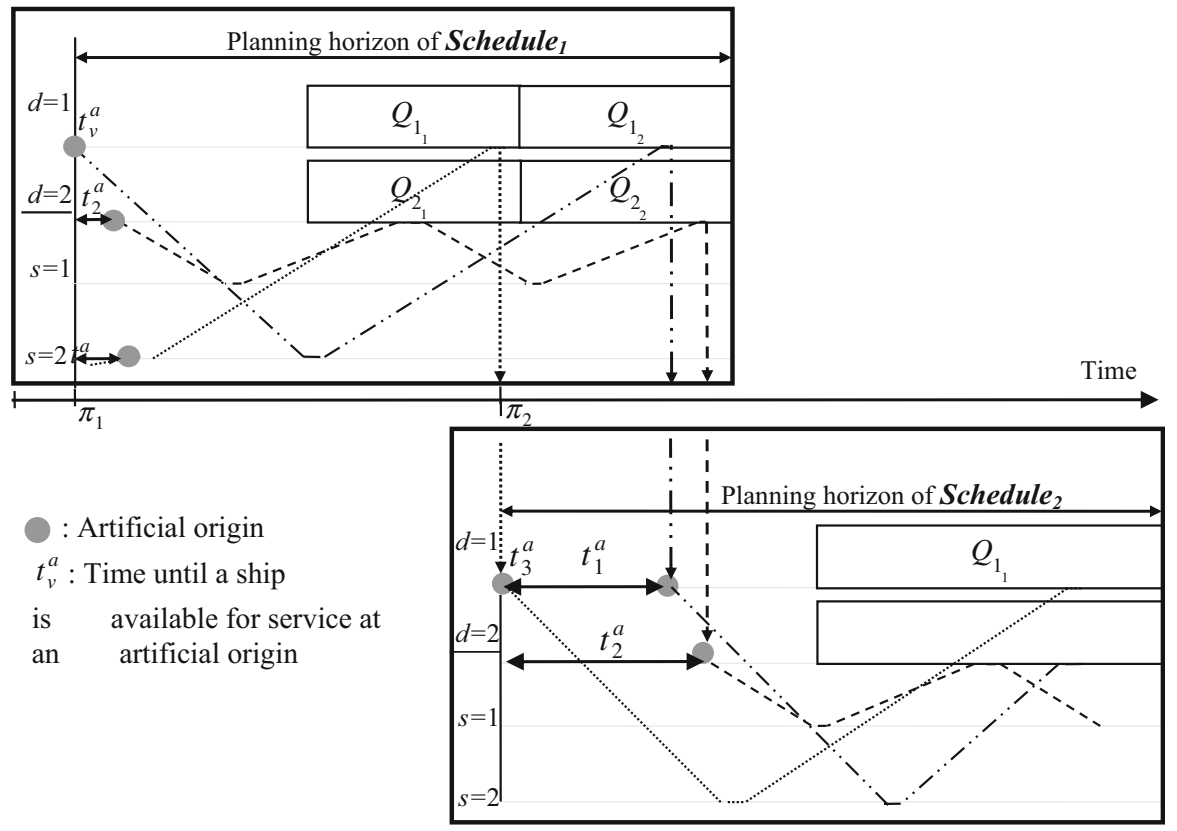

Fig. 2 Time-dependent schedules

on vessel positions the moment new demand is realized, but allows the en route vessels to continue till the end of the current leg. Clearly such a scheme is suitable where flexibility or adjustment in a schedule is allowed. In addition, we expect TDP2 to yield better results than TDP1, as it can exploit the newly available information, though it will require higher computational effort since the problem size increases. We next outline the distinct features of TDP2 in relation to TDP1, and invite the reader to consult "Appendix B" for the detailed algorithm.

TDP2 algorithm differentiates itself from TDP1 mainly in the way artificial origins are determined and assigned. In TDP2, artificial origins for the used ships can both be at the demand locations or the supply points depending upon when the demand for the next schedule is realized. As soon as this new demand is realized, the algorithm captures the next destination of en route vessels and sets them as artificial origins for the respective ships. It is important to note that the unfulfilled demand of the current schedule is terminated, and attached to the next schedule. Finally, the algorithm has the provision to allow any new demand location to be added to the new plan.

\section{Computational experiments}

In this section, we first describe a realistic size problem instance, which is then solved using the periodic requirement methodology introduced in the previous 
section. We also outline three special cases of the problem before providing extensive managerial insights.

\section{Problem instance}

We focus on the tanker fleet operation of Vela International Marine Limited (www. vela.ae), the wholly owned subsidiary of Saudi Aramco-the largest producer and exporter of crude oil. Vela is primarily responsible for deliveries to North America and to Europe, which is handled from its four ports in the Persian Gulf and the Red Sea. A total of twenty berths are available between the two ports in the Persian Gulf, and another four berths on the western side. Figure 3 depicts the primary oil routes from the two supply locations to the customer locations. Note that the Gulf of Mexico is 12,084 and 6,792 nautical miles, respectively, from the Persian Gulf and Red Sea, whereas the equivalent numbers for Europe are 6,393 and 3,803 nautical miles. Saudi Aramco (via Vela) runs most of its operations from the eastern ports, and aims to limit crude supply to less than $25 \%$ from the western ports due to upstream supply network restrictions.

For US demand, we consulted the weekly oil import figures from Saudi Arabia, made available by the Energy Information Administration, and assumed the European numbers to be approximately $25 \%$ of the US ones. Table 1 depicts the weekly crude oil import figures (in kilo tons) for a 3-month period, and forms the basis of our study. In an effort to mimic this setting, we consider three planning horizons each of 4 weeks in length, and assume that related demand information becomes available by the end of day 0,28 and 56, respectively. Figure 4 depicts the time when the three schedules start, the time span for the three planning horizons (i.e., 3 months), and the demand at the two locations over the planning period. For example, the first planning horizon starts at day 0 and ends at day 63, whereas the corresponding numbers are day 28 and day 91 for the second planning horizon (i.e., the one which includes July demand). Based on the available information, we assume that Vela owns twenty tankers, while ten additional tankers are available through chartering. While the capacities and travel times of the owned tankers are

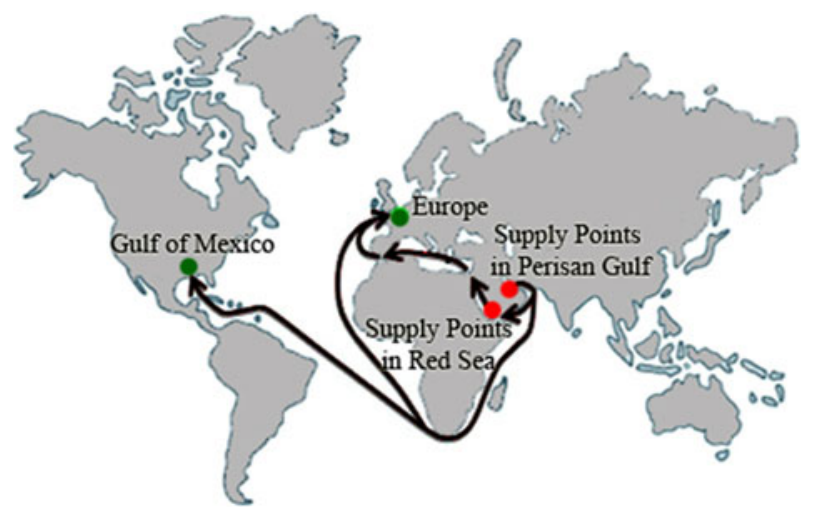

Fig. 3 Primary routes for vela (source: www.vela.ae) 
Table 1 Weekly crude oil imports (source: www.eia.gov)

\begin{tabular}{lcrlllll}
\hline & US & & & & Europe \\
& June & July & August & & June & July & August \\
\hline Week 1 & $1,174.6$ & $1,162.2$ & 860.4 & & 293.7 & 251.3 & 186.1 \\
Week 2 & 466 & 885.3 & 891 & & 100.8 & 191.4 & 192.7 \\
Week 3 & $1,000.8$ & 802.2 & 1,124 & & 216.4 & 173.5 & 243.1 \\
Week 4 & $1,140.2$ & $1,416.2$ & $1,157.4$ & & 246.6 & 306.2 & 250.3 \\
\hline
\end{tabular}

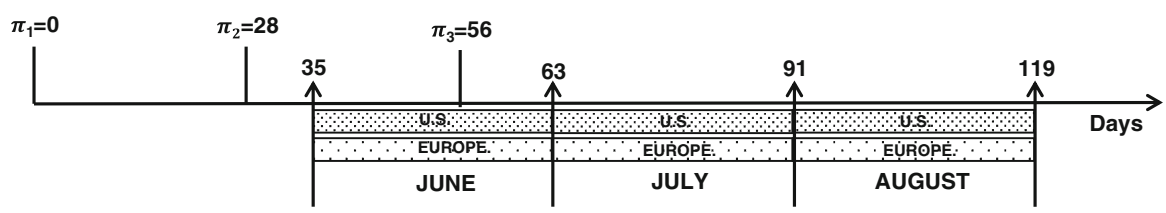

Fig. 4 Demand and planning horizons

available informations, we make use of the typical VLCC attributes to generate corresponding numbers for the ten chartered vessels (Table 2).

\section{Solution and discussion}

Before we can generate schedules for the given planning horizons, it is important to determine a suitable value for $\tau$ (i.e., maximum number of trips by a vessel during the planning horizon). This is an important parameter that will impact both the model size and the solution time, and should be just large enough to ensure that no feasible schedule is missed. We select $\tau$ based on the following simplified scheme:

LET: $\beta_{v}^{\prime}=\operatorname{Min}\left(\left[\beta_{v s d}\right]\right) \forall v \in V$ where $\beta_{v s d}=\left(t_{v s d}+t_{v d s}\right) \forall v \in V, s \in S, d \in D$

$$
\tau_{v}=\left\lceil P / \beta_{v}^{\prime}\right\rceil \quad v \in V
$$

(21) determines the shortest trip time by vessel $v$ between all pairs of $s$ and $d$, and back to $s$, which is then used to compute the maximum number of trips possible in (22). This can be done for each individual vessel, or we can use a single $\tau$ by dropping index $v$, and assuming $\tau_{v}=\left\lceil P / \beta^{\prime}\right\rceil$, where $\beta^{\prime}=\operatorname{Min}\left(\left[\beta_{v s d}\right]\right)$. A number of experimental runs were performed, and it was concluded that $\tau=2$ for each of the three planning horizons, which is in line with the observation in Hennig et al. (2011).

All instances of the managerial problems were solved using CPLEX 12.1 (IBM 2010), on a quad-core Intel-i7 computer, running a 64-bit Windows 7. The input files were generated using MATLAB 7.0.1.24704 (R14) (Mathworks 2004). Tables 3 and 4 depict the snapshot of the solution for the given planning horizon, when (PBM) was initialized using TDP1 and TDP2. Note that although we will investigate the impact 
Table 2 Vela fleet utilization under the two solution methods

\begin{tabular}{|c|c|c|c|c|c|}
\hline $\begin{array}{l}\text { Name of } \\
\text { vessel }\end{array}$ & $\begin{array}{l}\text { Crude oil carrying } \\
\text { capacity }(\mathrm{kT})\end{array}$ & $\begin{array}{l}\text { Speed (average) } \\
\text { (knots) }\end{array}$ & $\begin{array}{l}\text { Speed } \times \text { capacity } \\
\text { (scaled) }\end{array}$ & TDP1 & TDP2 \\
\hline Capricorn star & 317.129 & 14.8 & 4.7 & 1 & 1 \\
\hline Aries star & 317.129 & 14.4 & 4.6 & 1 & 1 \\
\hline Pisces star & 317.129 & 14.1 & 4.5 & 2 & 2 \\
\hline Leo star & 317.129 & 14.2 & 4.5 & - & - \\
\hline Pherkad star & 301.569 & 15.7 & 4.7 & 3 & 3 \\
\hline Markab star & 301.227 & 14.7 & 4.4 & 2 & 2 \\
\hline Polaris star & 301.569 & 14.9 & 4.5 & 1 & 2 \\
\hline Shaula star & 301.590 & 15.8 & 4.8 & 2 & 2 \\
\hline Mirfak star & 301.542 & 14.5 & 4.4 & 1 & 2 \\
\hline Alphard star & 301.862 & 15.7 & 4.7 & 3 & 2 \\
\hline Gemini star & 301.862 & 15.3 & 4.6 & 2 & 1 \\
\hline Suhail star & 301.862 & 15.8 & 4.8 & 3 & 3 \\
\hline Albutain star & 319.428 & 15.0 & 4.8 & 1 & 2 \\
\hline Sirius star & 319.428 & 16.0 & 5.1 & 2 & 2 \\
\hline Vega star & 319.428 & 14.7 & 4.7 & 1 & 1 \\
\hline Almizan star & 319.428 & 15.1 & 4.8 & 1 & 1 \\
\hline Janah star & 319.428 & 14.4 & 4.6 & 3 & 1 \\
\hline Saiph star & 318.000 & 15.0 & 4.8 & 1 & 2 \\
\hline Antares star & 391.400 & 14.8 & 5.8 & 2 & 2 \\
\hline Virgo star & 391.400 & 15.3 & 6.0 & 2 & 2 \\
\hline Chartered \# 1 & 326.667 & 15.3 & 5.0 & 2 & 1 \\
\hline Chartered \# 2 & 361.179 & 15.9 & 5.7 & 1 & 2 \\
\hline Chartered \# 3 & 313.616 & 14.4 & 4.5 & 2 & 2 \\
\hline Chartered \# 4 & 305.985 & 14.2 & 4.3 & 1 & 2 \\
\hline Chartered \# 5 & 332.298 & 15.1 & 5.0 & 2 & 1 \\
\hline Chartered \# 6 & 313.426 & 15.9 & 5.0 & 1 & 2 \\
\hline Chartered \# 7 & 315.172 & 14.0 & 4.4 & 2 & 1 \\
\hline Chartered \# 8 & 283.902 & 15.7 & 4.5 & 2 & 2 \\
\hline Chartered \# 9 & 343.129 & 14.1 & 4.8 & - & - \\
\hline Chartered \# 10 & 332.091 & 15.0 & 5.0 & 1 & 1 \\
\hline
\end{tabular}

stemming from the spatial distribution of tankers "Managerial insights", for this part of the analysis we assume that fifteen tankers have been randomly divided between the two supply sources, and the other fifteen between the two demand locations. In addition, we assume that the time to availability for these tankers range from zero to 10 days, where the range was determined after generating a number of scenarios such that the arrival of tankers for loading at supply sources was evenly distributed, and more importantly enabling each tanker sufficient time to make at least one feasible trip in the first planning horizon. The latter was based on the trip times that ranged from 20 days to 2 months. 
Table 3 Problem solution

\begin{tabular}{|c|c|c|c|c|c|c|}
\hline \multirow{2}{*}{$\begin{array}{l}\text { Planning } \\
\text { Periods }\end{array}$} & \multicolumn{3}{|l|}{ TDP1 } & \multicolumn{3}{|l|}{ TDP2 } \\
\hline & Cost $(\$)$ & Time (sec) & Gap (\%) & Cost $(\$)$ & Time (sec) & Gap (\%) \\
\hline June & $7,038,783$ & $16,686.2$ & 1.87 & $5,567,247$ & $16,686.2$ & 1.87 \\
\hline July & $7,530,499$ & 219.4 & - & $7,268,450$ & $39,905.9$ & 1.34 \\
\hline August & $7,912,029$ & $15,331.4$ & 1.09 & $9,289,108$ & $41,162.7$ & 1.10 \\
\hline Total & $22,481,311$ & 32,237 & & $22,124,805$ & 97,755 & \\
\hline
\end{tabular}

Table 4 Problem size

\begin{tabular}{llllll}
\hline Planning & \multicolumn{2}{l}{ TDP1 } & & \multicolumn{2}{l}{ TDP2 } \\
\cline { 2 - 3 } \cline { 5 - 5 } Periods & Constraints & 0/1 variables & & Constraints & 0/1 variables \\
\hline June & 16,876 & 8,580 & 16,876 & 8,580 \\
July & 16,876 & 8,580 & 17,360 & 8,820 \\
August & 16,876 & 8,580 & 17,360 & 8,820 \\
\hline
\end{tabular}

In an effort to evaluate the economic performance of a vessel vis-à-vis other vessels in terms of their speed and capacity, we compared ship usage with speed, capacity and speed $\times$ capacity (Fig. 5). We believe such information is vital in facilitating better fleet management as it reflects the relative performance of a vessel, and consequently is a good indicator of the expected utilization. However, it is important to note that ship usage is also a function of the demand constraint [i.e., Eq. (2)] and the ship location since a tanker that is economically less preferred may still be used if nothing better is available, which in turn will impact the analysis. But, in general, it was noticed that tankers with higher speeds were preferred, while the impact of capacity was less pronounced under both initialization techniques. For example, fastest ships such as Pherkad Star, Alphard Star and Suhail Star were used thrice, while Leo Star was never used. This effect is expected as extremely large lead time is a major issue. On the other hand, it was noticed that some of the largest ships such as Janah Star and Antares Star also had higher usages, which could have been driven by the need to realize the economies of scale by sending fewer ships, if possible.

For the first planning horizon (i.e., June), both initializations resulted in the same amount of computing time to arrive at exactly the same solution with identical gap from the best bound indicated in the CPLEX (Table $3^{3}$ ). This was because, for both TDP1 and TDP2, artificial origins and time to availability (i.e., $t_{v}^{a}$ ) for all tankers are

\footnotetext{
3 Solution times with LP-relaxation is in fractions of a second $(\sim 0.15 \mathrm{~s})$ for all the problems, however, solution itself is not reported. This is because the binary variables can be satisfied by just varying $w_{v d_{i}}^{j}$ in constraints set (3), and leaving all $x_{v s d}^{j}=0$ and $y_{v d s}^{j}=0$. Only the binary variables representing empty vessels returning from artificial origins are assuming a value of 1 [i.e., in constraints set (8)], which implies that schedules are generated only for the movement from artificial origins to the supply sources and nothing beyond. This precludes any meaningful comparison with the solution without relaxation.
} 


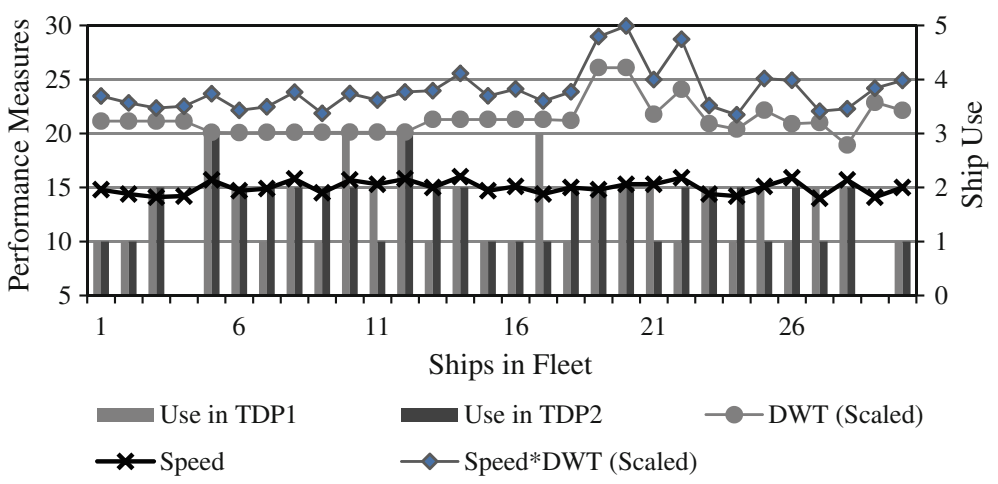

Fig. 5 Vessel utilization (the ship numbers follow the same order as in Table 2. For example, ship 1 refers to Capricorn star)

exactly the same. Model size is presented in Table 4 depicting the number of constraints (excluding variable type constraints) and the number of binary variables in the corresponding models. TDP2 results in lower cost, which stems from the cancelation of seven trips as soon as the July demand information is received. The unfulfilled demand, from June, was appended with the next planning horizon, where the costs are more comparable (i.e., $\$ 7,530,499$ vs. $\$ 7,268,450$ ). It is important that the total cost for the month of July was $\$ 8,394,388$, which reduced to just over seven million once demand for August necessitated canceling six trips. Clearly the demands associated with the canceled trips have to be met before the end of the planning horizon, which explains the rather high total cost for the month of August. The need to meet demand before the end of the planning horizon is also the reason behind the increase in the number of variables as well as constraints, since the number of demand periods increased from four to six. It appeared that initializing (PBM) using TDP2 may result in savings of around $1.6 \%$ compared to the solution returned by TDP1 (i.e., $\$ 22.5$ million), although the computation time for the latter technique is preferable. It should be evident that more effective exploitation of new information, at the start of each planning horizon, brings about adjustments in tanker slates, thereby driving down costs.

For the given problem instances, computation times varied across the planning horizons for both types of initializations. Slow convergence-without significant improvement - was noticed when the gap was within $2 \%$, which motivated us to terminate the runs in around $4 \mathrm{~h}$ for TDP1, and between 4 and $12 \mathrm{~h}$ for TDP2.

For TDP1, the problem size did not change across the three planning horizons, the computation time did since it depends on the location of the vessels. For example, for the July planning horizon, 14 vessels were available at supply sources as artificial origins with an average time to availability of 9.4 days (vs. 15 vessels and 4.7 days for June). This is important since computational flexibility (and hence time) for any planning horizon depends on: the number of vessels present at the supply sources; time to availability; and number of vessels that can return from demand points in time. Clearly, scenarios with large values of the above attributes will necessitate longer computing time than the ones without, since the search space 
(and hence the number of feasible alternatives) will be bigger. Finally, computation time for August was significantly more than that for July, though both planning horizons had exactly 14 vessels at the supply sources. On further investigation it was noticed that in addition to the average time to availability (viz. 12.8 days), the exact distribution of vessels between the two supply sources (and the supply quota at the western ports) was driving the computation time. For instance, all the 14 vessels are located on the western ports in August, while the number was 12 in July. Since western ports have only four berths and are subject to supply quotas, most of these vessels have to be routed to the eastern ports, and this number will be at least two more in the month of August. It should be clear that these re-positioned vessels are going to compete with the ones returning from demand locations in time for being considered for scheduling. Although such flexibility (i.e., number of vessels available for scheduling) is preferred since it can result in lower cost, it can involve significant computation time. While higher flexibility did result in lower cost for June, it was not the case for August since vessel re-positioning (and idling) negated the savings. In an effort to further investigate the role of vessel location and time to availability, relevant parametric analysis was conducted and the resulting insights are reported in "Managerial insights".

On the other hand, for TDP2 a straightforward analysis of time across the planning horizons is not meaningful since the model size changes as a result of appending unfulfilled demand requirements. For example, two demand periods (i.e., weeks) are appended to the planning horizons in July and August, which resulted in a six-period problem with additional variables and constraints (240 binary and 484 constraints). For both planning horizons, computations were terminated given an increasingly slower convergence and running for very long hours (i.e., over $11 \mathrm{~h}$ in these cases). Given the combinatorial nature of the problem, in general, the computation time will increase with growth in problem size.

\section{Special cases}

Not all crude oil suppliers have the luxury of multiple ports (or supply points). For example, Kuwait Petroleum Corporation (KPC) meets all its supply requirements from a single port, whereas Saudi Aramco-with two ports-primarily relies on its eastern ports to meet demand. The aforementioned implies that while KPC may experience port capacity issues, it need not worry about supply quota, which definitely needs to be considered when developing the transportation plan for Saudi Aramco.

In an effort to incorporate different capacity and supply quota scenarios at supply points, we outline three special cases of (PBM). First is (PCC), which excludes constraints (19), since only port capacity constraints are pertinent. Second is (SQC), which excludes constraints (14)-(18), since only supply distribution quota constraints are relevant. Third is (NSC), which excludes constraints (14)-(19), since neither port capacity nor supply quota constraints are required.

The realistic problem instance, after appropriate modifications, was solved for the three special cases and the corresponding results are depicted in Table 5, while the problem sizes are presented in Table 6 wherein we once again notice an increase in 
the size of the problem under TDP2. As expected, (NSC) yields the most inexpensive solution, since the resulting formulation is the least constrained. On the other hand, the introduction of supply quota constraints [i.e., in (PBM) and (SQC)] resulted in significant cost increase, since more vessels have to travel longer distances to adhere to the supply quota constraints. It is possible to conclude that the special cases with fewer constraints require far less computing time, and that the resulting solutions are either optimum or very close to optimum. For instance, with TDP2 initialization, the average computing time for the entire planning horizon goes up from $11,576 \mathrm{~s}$ for (NSC) to over 30,875 s for (SQC). It should also be noted that TDP2 outperforms TDP1 in the presence of fewer constraints, as there is more flexibility to schedule vessels in an effort to exploit new information. For example, cost savings increase from $1.36 \%$ for (SQC) to $5.16 \%$ for (NSC). Like (PBM), vessels with higher speeds are preferred (as discussed in "Solution and discussion"), and this phenomenon is more pronounced when using TDP2 for initialization since the flexibility increases. A strong similarity in fleet utilization, especially with TDP1 initialization, was noticed between (NSC) and (PCC) cases, and (SQC) and (PBM) instances. This was not unexpected, since the absence of any supply quota constraints encouraged the use of western ports (i.e., Red Sea) and a greater number of preferred vessels. For instance over the 3-month planning horizon, only 20 and 22 of the available vessels in the fleet were used in the (NSC) and (PCC) cases, whereas the numbers are 26 and 28, respectively, for (SQC) and (PBM). With TDP2, the fleet utilization figures for (NSC) and (PCC) increased to 24 each, while it remained unchanged for the other two. This increase in utilization is also reflected in comparative cost savings between TDP2 and TDP1 for the two cases, and they are 5.16 and $5.03 \%$, respectively.

Table 5 Snapshot of the special cases

\begin{tabular}{|c|c|c|c|c|c|c|c|}
\hline \multirow{2}{*}{$\begin{array}{l}\text { Special } \\
\text { Cases }\end{array}$} & \multirow{2}{*}{$\begin{array}{l}\text { Planning } \\
\text { periods }\end{array}$} & \multicolumn{3}{|l|}{ TDP1 } & \multicolumn{3}{|l|}{ TDP2 } \\
\hline & & Cost (\$) & $\begin{array}{l}\text { Time } \\
(\mathrm{sec})\end{array}$ & Gap & Cost (\$) & $\begin{array}{l}\text { Time } \\
(\mathrm{sec})\end{array}$ & Gap \\
\hline \multirow[t]{4}{*}{ NSC } & June & $5,984,191$ & 19.7 & - & $4,139,822$ & 19.7 & - \\
\hline & July & $6,265,297$ & 3.8 & - & $5,342,505$ & $31,005.9$ & $1.97 \%$ \\
\hline & August & $6,231,035$ & 2.8 & - & $8,044,120$ & $3,704.9$ & - \\
\hline & Total & $18,480,523$ & 26 & & $17,526,447$ & 34,731 & \\
\hline \multirow[t]{4}{*}{ PCC } & June & $6,004,546$ & $3,527.4$ & - & $4,138,025$ & $3,527.4$ & - \\
\hline & July & $6,298,237$ & 62.7 & - & $5,379,355$ & $50,418.2$ & $2.90 \%$ \\
\hline & August & $6,363,484$ & 10.4 & - & $8,210,283$ & $31,963.1$ & $1.09 \%$ \\
\hline & Total & $18,666,267$ & 3,601 & & $17,727,663$ & 85,909 & \\
\hline \multirow[t]{4}{*}{ SQC } & June & $7,026,602$ & $15,544.8$ & $1.16 \%$ & $5,351,212$ & $15,544.8$ & $1.16 \%$ \\
\hline & July & $7,463,264$ & 6.3 & - & $7,112,660$ & $45,472.5$ & $1.69 \%$ \\
\hline & August & $7,830,326$ & 567.1 & - & $9,552,982$ & $31,608.7$ & $0.54 \%$ \\
\hline & Total & $22,320,192$ & 16,118 & & $22,016,854$ & 92,626 & \\
\hline
\end{tabular}


Table 6 Problem size

\begin{tabular}{|c|c|c|c|c|c|}
\hline \multirow[t]{2}{*}{ Special cases } & \multirow[t]{2}{*}{ Planning periods } & \multicolumn{2}{|l|}{ TDP1 } & \multicolumn{2}{|l|}{ TDP2 } \\
\hline & & Constraints & $0 / 1$ variables & Constraints & $0 / 1$ variables \\
\hline \multirow[t]{3}{*}{ NSC } & June & 1,932 & 1,020 & 1,932 & 1,020 \\
\hline & July & 1,932 & 1,020 & 1,932 & 1,260 \\
\hline & August & 1,932 & 1,020 & 1,932 & 1,260 \\
\hline \multirow[t]{3}{*}{ PCC } & June & 16,874 & 8,580 & 16,874 & 8,580 \\
\hline & July & 16,874 & 8,580 & 17,358 & 8,820 \\
\hline & August & 16,874 & 8,580 & 17,358 & 8,820 \\
\hline \multirow[t]{3}{*}{ SQC } & June & 1,934 & 1,020 & 1,934 & 1,020 \\
\hline & July & 1,934 & 1,020 & 1,934 & 1,260 \\
\hline & August & 1,934 & 1,020 & 1,934 & 1,260 \\
\hline
\end{tabular}

At Supply Points

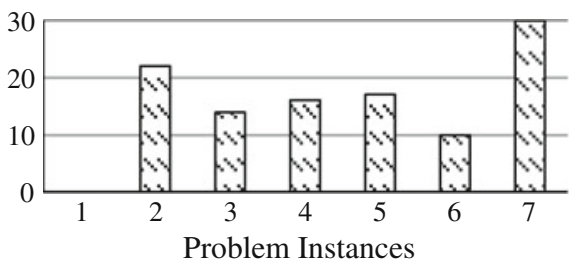

At Demand Points

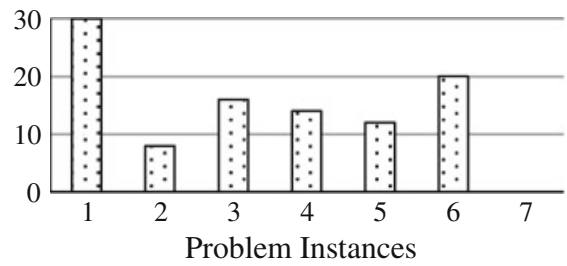

Fig. 6 Number of vessels

Managerial insights

In an effort to understand how flexibility affects cost and computing time, we varied the artificial origins and the time to availability (i.e., $t_{v}^{a}$ ) for problem instances involving four requirement periods (i.e., 4 weeks in a month). To assess the impact of "artificial origin" with fixed $t_{v}^{a}$, seven problem instances were generated wherein first and seventh were the two extreme cases, and vessels were randomly assigned between the supply sources and demand locations in the other five (Fig. 6). It is important that first instance represents the least flexible situation since the artificial origins for all vessels are at demand locations, and seventh the most flexible since the entire fleet is available at the supply sources.

Although each of the seven problem instances was solved using (PBM), and the three special cases, for expositional purposes and also for brevity we report only on the two extreme settings [i.e., (PBM) and (NSC)]. Figure 7 depicts the values for the two relevant settings, and we can report that a similar pattern was noticed for (PCC) and (SQC) cases. In general, the cost increased when more vessels were located at demand points, since longer distances have to be covered before the vessels could be used to make trips. On the other hand, the computation time increased when more vessels were present at the supply sources, since this added more flexibility to 

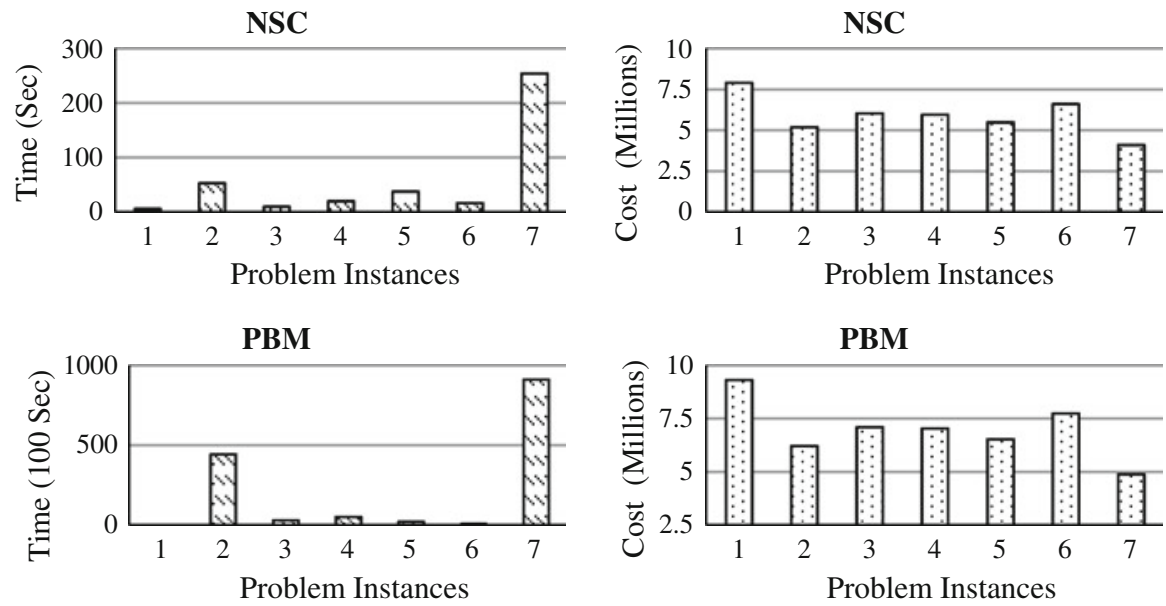

Fig. 7 Sensitivity to artificial origins

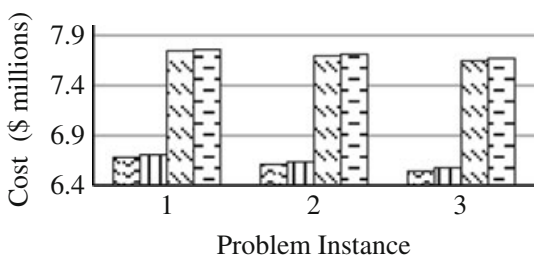

图 NSC III PCC 』SQC $\mathrm{P}$ PBM

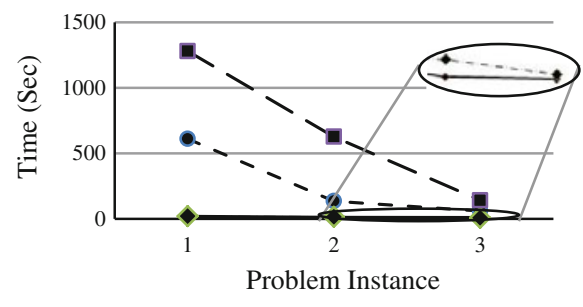

$\longrightarrow-\mathrm{NSC}-\bullet-\mathrm{PCC}-\cdots \cdot \mathrm{SQC} \longrightarrow-\mathrm{PBM}$

Fig. 8 Sensitivity to $t_{v}^{a}$

the model. Finally, as noted earlier, the least constrained setting does result in a better solution for every problem instance.

Next, we considered three new problem instances where the artificial origins were fixed at the supply sources but $t_{v}^{a}$ was varied. Once again each problem instance was solved using (PBM), and the three special case formulations. For the first problem instance, systematic randomness is introduced into the $t_{v}^{a}$ as follows: the first vessel is assigned a random $t_{v}^{a}$, then a 0.5-day separation is added to the next vessel and so on until the tenth vessel. To avoid unrealistic values of $t_{v}^{a}$, the pattern restarts after ten vessels. Similarly the second and third problem instances are generated using 1.0- and 1.5-day separation, and the snapshot of the result is depicted in Fig. 8. This systematic randomness will facilitate investigating the impact of delayed availability of vessels for the three problem instances. It appears that cost decreases with increase in $t_{v}^{a}$, since the waiting time for the ships at the supply sources goes down. In addition, the computing time goes down with increase in $t_{v}^{a}$, since now vessel availability is staggered which limits flexibility and hence time needed to reach a solution. 


\section{Decomposition heuristic}

Although realistic size problem instances in the previous section were solved using a commercial solver, it is easy to see that the combinatorial structure of the problem could render much larger instances (i.e., more variables and constraints) intractable. In this section, we propose a heuristic to solve such large scale problems. The basic idea in this heuristic is to break a large problem into a number of smaller subproblems, which span across timelines and are solved sequentially as timedependent periodic planning problems. The resulting sub-problems are initialized based on the ending positions of vessels from the previous one (as depicted in Fig. 2). Figure 9 depicts an illustrative example with four periods (or weeks), which has been broken into two sub-problems.

In Fig. 9, the start time for the sub-problems is based on earliest tanker availability in that sub-problem. Secondly, the heuristic also calls for defining a decision rule to include/exclude vessels. For example, if a vessel finishes delivery at time $P_{1}$ to the US, it may not be able to return to the supply port by $P_{2}$, and hence should be excluded from model formulation of the second sub-problem (i.e., Subproblem $_{2}$ ). In addition, vessels that can return from demand locations feasibly but are not used for additional trips during the next sub-problem should also be excluded, since they will result in inflated costs. It is important to note that a tanker that has been excluded from the current sub-problem will be a part of the subsequent sub-problem, with the existing location and time becoming the starting point for consideration in the subsequent sub-problem. Doing so would not only ensure tanker movement continuity but also enable appropriate accounting of all costs, namely waiting and traveling. "Appendix C" provides the summary of steps, including the exclusion and inclusion rules, needed to implement the proposed heuristic.

In an effort to evaluate the efficacy of the proposed heuristic, five of the seven random problem instances_introduced "Managerial insights"—-were solved. Each problem has a four-week planning horizon, and excludes the two extreme cases (i.e., when vessels are either at the supply or demand locations). Please note that the model sizes for all four cases are exactly as those given for the month of June (i.e., Tables 3, 4, 5 and 6). Table 7 depicts the performance of the proposed heuristic in comparison to the solution methodology outlined in "Solution methodology", while Table 8 lists the actual costs and computing times when the proposed heuristic is not

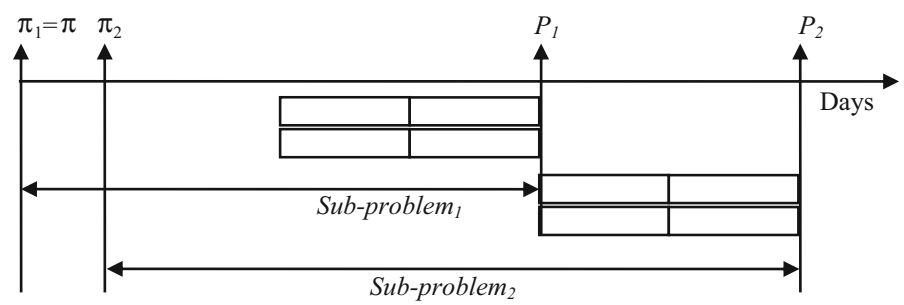

Fig. 9 Four-period problem with two sub-problems 
Table 7 Performance of heuristic method

\begin{tabular}{|c|c|c|c|c|c|c|c|c|}
\hline \multirow[t]{2}{*}{ Instances/models } & \multicolumn{4}{|c|}{$\%$ Cost increase } & \multicolumn{4}{|c|}{$\%$ Time savings } \\
\hline & NSC & PCC & SQC & PBM & NSC & PCC & SQC & PBM \\
\hline 1 & 1.12 & 2.11 & 3.03 & 3.13 & 96.65 & 99.44 & 99.94 & 99.60 \\
\hline 2 & 1.26 & 1.79 & 1.90 & 2.60 & 84.78 & 97.84 & 99.85 & 98.21 \\
\hline 3 & 1.06 & 1.89 & 2.15 & 2.24 & 93.76 & 97.46 & 99.95 & 96.22 \\
\hline 4 & 1.83 & 1.40 & 1.80 & 1.99 & 95.50 & 98.50 & 99.92 & 96.31 \\
\hline 5 & 1.96 & 1.95 & 3.18 & 3.20 & 73.31 & 87.57 & 73.40 & 85.42 \\
\hline Average & 1.45 & 1.83 & 2.41 & 2.63 & 88.80 & 96.16 & 94.61 & 95.15 \\
\hline
\end{tabular}

Table 8 Performance without the heuristic

\begin{tabular}{|c|c|c|c|c|c|c|c|c|}
\hline \multirow[t]{2}{*}{ Instances/models } & \multicolumn{4}{|c|}{ Cost (in millions) } & \multicolumn{4}{|c|}{ Time (in min) } \\
\hline & NSC & PCC & SQC & PBM & NSC & PCC & SQC & PBM \\
\hline 1 & 5.195 & 5.217 & 6.198 & 6.212 & 0.88 & 281.63 & 601.04 & 739.50 \\
\hline 2 & 6.038 & 6.070 & 7.065 & 7.106 & 0.16 & 15.75 & 76.12 & 47.64 \\
\hline 3 & 5.970 & 5.993 & 7.030 & 7.047 & 0.34 & 20.43 & 228.66 & 83.33 \\
\hline 4 & 5.497 & 5.517 & 6.526 & 6.533 & 0.63 & 19.14 & 122.13 & 34.54 \\
\hline 5 & 6.610 & 6.635 & 7.694 & 7.713 & 0.28 & 2.31 & 0.27 & 10.39 \\
\hline Average & 5.862 & 5.886 & 6.902 & 6.922 & 0.457 & 67.852 & 205.6 & 183.1 \\
\hline
\end{tabular}

used. Although the proposed heuristic exhibits cost increases for all model types, the average increment is largest for the most constrained model (i.e., 1.45 vs. $2.63 \%$ ). On the other hand, the average time to arrive at a solution decreases significantly for all models. Note that the largest average solution time for the given problem set, without the heuristic, was close to $4 \mathrm{~h}$ (SQC) with a maximum of over $12 \mathrm{~h}(\mathrm{PBM})$; a time reduction in order of 73-99\% with such long solution times is certainly a notable performance factor for the heuristic. This is an important result, since the proposed heuristic can help determine a good quality solution-within a reasonable amount of time-for large scale problem instances that could be potentially intractable. It should be clear that selecting the number of sub-problems, and its impact on solution quality and time, are perhaps the most important determinant of heuristic effectiveness. Unfortunately, the appropriate number of sub-problems can be determined only by iteratively solving the given problem till a solution is reached.

\section{Conclusion}

In this paper, we have proposed a novel analytical scheduling approach for crude oil tankers, which includes two distinct solution methodologies-inspired by the 
pervasive nature of maritime crude oil transportation. The proposed approach was motivated by a desire to exploit the problem characteristics by incorporating periodic demand requirements directly into the model, which is dissimilar to the popular approach in the literature, and is used in only two works wherein the cargo set is used as an input. The analytical framework was tested on realistic size problem instances generated using the maritime infrastructure of Vela International Marine Limited, which were further analyzed to gain managerial insights. It was noticed that artificial origins, spatial distribution of tankers and their time to availability are important to the solution quality and the computing time. To cater to varying scenarios, three special cases of the analytical framework were also solved and compared to the general case situation. Introduction of supply quota and port capacity constraints resulted in more expensive solutions, and also required larger computing time. A decomposition-heuristic for very large problem instances was outlined, and then used to solve random problem instances. It was demonstrated that significant reduction in computing time is possible by accepting a marginally more expensive solution.

This work has a threefold contribution: first, it proposes a split delivery scheduling framework for a global crude oil supplier, where the natural demand structure is used as an input; second, it develops two time-dependent sequential planning approaches for scheduling crude oil tankers; and third, it suggests a simplification technique to tackle large and potentially intractable problem instances. Directions for future research include several generalizations such as problems involving multiple grades of crude oil and various forms of VLCC chartering. Integration of planned maintenance decisions (requiring planned halting of ship operations to complete dry-docking for major maintenance) is another area. Planned maintenance ensures high ship reliability and availability while minimizing unexpected breakdowns and the associated high costs resulting from missed or delayed orders, rapid transportation of parts and personnel to distant ship locations, etc. (Deris et al. 1999). Out of various types of maintenance strategies [e.g., preventive, condition based, reliability centered (Duffuaa et al. 1999; Siddiqui and Ben-Daya. 2009)] preventive maintenance is scheduling based, i.e., decisions based on time or traveled distance. Integration of crude oil deliveries as well as preventive maintenance is important as VLCCs operates on a busy schedule while maintenance scheduling requires assignment of specified time slots which is based on maintenance requirements, availability of parts and staffing issues, etc., and thus requires due consideration.

Acknowledgments This research has been supported in part by a grant from the National Sciences and Engineering Research Council of Canada (Grant \# 312936). The second author is a member of the Interuniversity Research Center on Enterprise, Network Logistics and Transportation (CIRRELT) and acknowledges the research infrastructure provided by the Center. The authors are grateful to the three anonymous referees, whose constructive feedback was helpful in improving the paper. 


\section{Appendix}

\section{Appendix A: Algorithm for TDP1}

Please note that a backward arrow (i.e., $\leftarrow$ ) on a variable or parameter indicates association with the previous schedule, a forward arrow (i.e., $\rightarrow$ ) denotes carrying the partial set forward to the new schedule, and no arrow indicates elements of the new schedule. The steps of the algorithm is as follows:

1. $n \leftarrow$ New planning horizon index;

2. IF $n=1$;

SET parameters (cost, time, demand \& supply: as listed in Section 4.1) for Schedule $_{1}$;

GENERATE \& SOLVE PBM (using a solver like CPLEX) to find Schedule ; $_{1}$ ELSE GOTO 3;

3. // Initializing model parameters for Schedule $_{n>1}$;

//Initializing vessels' artificial origin and time to availability;

$\vec{V} \leftarrow$ Set of vessels used in Schedule $_{n-1}$ carrying forward to Schedule ${ }_{n}$;

3.1 // Determining new artificial origins for the vessels in $\vec{V}$;

$A \leftarrow \phi ; / /$ Starting with a null set;

// Artificial origin is the end point of a vessel $v$ in Schedule $_{n-1}$ (i.e. a location conditional on its movement (or visits))

3.2

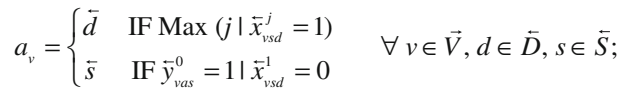

$A \leftarrow \cup\left\{a_{v}\right\}$;//appending all new artificial origins in set $A$;

3.3 // Determining $t_{v}^{a}$, relative to $\pi_{n}$;

$$
t_{v}^{a} \leftarrow\left\{\begin{array}{cc}
\pi_{n-1}+\operatorname{Max}\left(\bar{f}_{v}^{j}\right)-\pi_{n} & \operatorname{IF} \bar{x}_{v s d}^{1}=1 \\
0 & \operatorname{IF} \bar{x}_{v s d}^{1}=0
\end{array} \quad \forall v \in \vec{V}, d \in \bar{D}, s \in \bar{S}, ;\right.
$$

3.4 $\hat{V} \leftarrow$ The set of new vessels available for service; $V \leftarrow \vec{V} \cup \hat{V}$;

3.5 SET artificial origin and time to availability for vessels in $\hat{V}$;

3.6 SET parameters (cost, time, demand \& supply: as listed in Section 4.1) for Schedule S $_{n}$,

3.7 GENERATE \& SOLVE PBM (using a solver like CPLEX) to find Schedule $e_{n}$;

4. Repeat 1-4 when new orders are received; 


\section{Appendix B: Algorithm for TDP2}

\section{The steps of the algorithm is as follows:}

1. $N \leftarrow$ Next planning horizon index;

2. IF $n=1$;

SET parameters (cost, time, demand \& supply: as listed in Section 4.1) for Schedule S $_{1}$

GENERATE \& SOLVE PBM (using a solver like CPLEX) to find Schedule S $_{1}$; ELSE GOTO 3;

3. // Initializing model parameters for Schedule $_{n>1}$;

//Initializing vessels' artificial origin and time to availability;

3.1 $\vec{V} \leftarrow$ Set of vessels used in Schedule $_{n-1}$ carrying forward to Schedule $e_{n}$;

// Determining new artificial origins for the vessels in $\vec{V}$;

$A \leftarrow \phi$; / Starting with a null set;

// Artificial origin of a vessel $v$ is a point where it ends its $1^{\text {st }}$ leg that crosses $\pi_{n}$ in Schedule $_{n-1}$ (i.e. a location conditional on its movement (or visits))

$$
a_{v}=\left\{\begin{array}{ll}
\bar{d} & \operatorname{IF} \operatorname{Min}\left(j \mid \bar{x}_{v s d}^{j}=1 \&\left(\pi_{n-1}+\bar{f}_{v}^{j}\right) \geq \pi_{n} \&\left(\pi_{n-1}+\bar{e}_{v}^{j}\right)<\pi_{n}\right) \\
\bar{s} & \operatorname{IF} \operatorname{Min}\left(j \mid \bar{x}_{v s d}^{j}=1 \&\left(\pi_{n-1}+\bar{e}_{v}^{j}\right) \geq \pi_{n}\right) \\
\bar{s} & \operatorname{IF} \bar{y}_{v a s}^{0}=1 \mid \bar{x}_{v s d}^{1}=0
\end{array} \quad \forall v \in \vec{V}, d \in \bar{D}, s \in \bar{S} ;\right.
$$

$A \leftarrow \cup\left\{a_{v}\right\}$;//appending all new artificial origins in set $A$;

// Determining $t_{v}^{a}$, relative to $\pi_{n}$;

// At first we define $\zeta_{v}^{j}$, for the smallest index $j$ of the used trips by a vessel $v$ satisfying condition, as:

$/ / \pi_{n}$ is crossed by the loaded leg of trip $j, \zeta_{y}^{j} \leftarrow \overleftarrow{f}_{j}^{j}$;

$/ / \pi_{n}$ is crossed by the return leg of trip $j, \zeta_{v}^{j} \leftarrow \hat{e}_{v}^{j}-\leftarrow_{v}^{j}$;

$/ / \pi_{n}$ is crossed during idling at $s$ or vessel not used, $\zeta_{v}^{j} \leftarrow 0$; //Mathematically:

$$
\begin{aligned}
& \zeta_{v}^{j}= \begin{cases}\bar{f}_{v}^{j} & \operatorname{IF~Min~}\left[j \mid \bar{x}_{v s d}^{j}=1 \&\left(\pi_{n-1}+\bar{f}_{v}^{j} \geq \pi_{n}\right) \&\left(\pi_{n-1}+\bar{e}_{v}^{j}<\pi_{n}\right)\right] \\
\bar{e}_{v}^{j}-\overleftarrow{b}_{v}^{j} & \operatorname{IF~Min~}\left[j \mid \bar{x}_{v s d}^{j}=1 \&\left(\pi_{n-1}+\bar{e}_{v}^{j} \geq \pi_{n}\right) \&\left(\pi_{n-1}+\left(\bar{e}_{v}^{j}-\check{b}_{v}^{j}\right) \geq \pi_{n}\right)\right] \\
0 & \operatorname{IF~Min~}\left[j \mid \bar{x}_{v s d}^{j}=1 \&\left(\pi_{n-1}+\bar{e}_{v}^{j} \geq \pi_{n}\right) \&\left(\pi_{n-1}+\left(\bar{e}_{v}^{j}-\bar{b}_{v}^{j}\right)<\pi_{n}\right)\right] \\
0 & \operatorname{IF} \bar{x}_{v s d}^{1}=0\end{cases} \\
& t_{v}^{a}=\pi_{n-1}+\zeta_{v}^{j}-\pi_{n} \quad \forall v \in \vec{V} ;
\end{aligned}
$$

3.3 // All remaining used trips are terminated in Schedule S $_{n-1}$.

//All unsatisfied demand $Q_{d_{i}}^{\prime}$ is appended with the Schedule $e_{n}$. Determining $Q_{d_{i}}^{\prime}$ :

$\vec{D} \leftarrow \phi$; // Starting with a null set of demand points with unsatisfied demand

$\forall a_{v}$ in step $3.1, \hat{j}_{v} \leftarrow j ; / / j$ is the corresponding trip index;

// Let $T_{v}^{j}$ be an array of indices on $\overleftarrow{w}_{v d_{i}}^{j}$ for vessel $v$ and trip $j$; Then

IF $a_{v}=\bar{s}$ in step $3.2 ; \vec{D} \leftarrow \cup\{d\} \& T_{v}^{j} \leftarrow[d, i] \forall \bar{w}_{v d_{i}}^{j}=1, v \in V, j \geq \hat{j}_{v}$;

IF $a_{v}=\overleftarrow{d}$ in step 3.2; $\vec{D} \leftarrow \cup\{d\} \& T_{v}^{j} \leftarrow[d, i] \forall{\bar{w}_{v d} d_{i}}^{j}=1, v \in V, j>\hat{j}_{v}$;

$\bar{\delta}_{d}^{i} \leftarrow$ Surplus value in constraint (2) in Schedule Scl $\forall d \in \vec{D}, i\left(d, T_{v}^{j}\right)$;

$Q_{d_{i}}^{\prime} \leftarrow \sum_{v \mid \exists T_{v}^{j}=[d, i]} C_{v}-\grave{\delta}_{d}^{i} \quad \forall d \in \vec{D}, i\left(d, T_{v}^{j}\right)$

3.4 $\hat{D} \leftarrow$ Set of demand points corresponding to new orders/adjustment; SET $D=\vec{D} \cup \hat{D}$;

3.5 UPDATE (using $Q_{d_{f}}^{\prime}$ ) and SET all the new periodic requirements for Schedule ${ }_{n}$;

3.6 Subtract all costs of the terminated legs from the objective function value in Schedule $_{n-1}$;

3.7 $\hat{V} \leftarrow$ The set of new vessels available for service; $V \leftarrow \vec{V} \cup \hat{V}$;

3.8 SET artificial origin and time to availability for vessels in $\hat{V}$;

3.9 SET the remaining parameters (cost, time, demand \& supply: as listed in Section 4.1) for Schedule $_{n}$;

3.10 GENERATE \& SOLVE PBM (using a solver like CPLEX) to find Schedule ;

4. Repeat 1-4 when new orders are received; 


\section{Appendix C: Algorithm for time-based decomposition heuristic}

The steps of the algorithm is as follows:

// Original problem decomposed into (time-based) $M$ sub-problems to be solved sequentially

1. $M \leftarrow$ The number of sub-problems to the original problem, where $M \leq \operatorname{Max}(i)$;

$\Pi \leftarrow$ The start time of the original problem;

$P \leftarrow$ The planning horizon of the original problem;

$m \leftarrow 1$;// setting the sub-problem index value to 1 ;

2. IF $m=1$;

$\Pi_{l} \leftarrow \Pi$; //The start time of Sub-problem ${ }_{l}$;

$P_{1} \leftarrow$ Horizon spanning from $\Pi_{1}$ to the last requirement period included in the Sub-problem ; $_{1}$;

SET time and cost parameters (as listed in Section 4.1) for Sub-problem ; $_{1}$;

GENERATE \& SOLVE PBM (using a solver like CPLEX) to find Schedule for Sub-problem ; $_{\text {; }}$ ELSE GOTO 3;

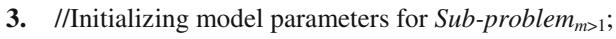

3.1 // Determining sub-problem start time and planning horizon;

Use steps 3.1-3.3 in TDP1 algorithm to find $a_{v}, t_{v}^{a} \forall v \in V$;

$\Pi_{m} \leftarrow \operatorname{Min}\left(t_{v}^{a}\right)$

$P \leftarrow$ Horizon spanning from $\Pi_{m}$ to the last requirement period included in the Sub-problem ; $_{m}$;

// Determining vessel availability in Sub-problem ${ }_{m}$ by using exclusion-inclusion rules;

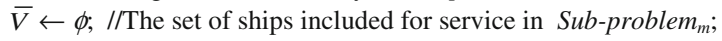

$\underline{V} \leftarrow \phi$; //The set of ships excluded from service in Sub-problem ${ }_{m}$;

IF vessel $v \in V \&$ not used in any earlier Sub-problem,

THEN $\bar{V} \leftarrow \cup\{v\} ; / /$ include unused vessels;

//Following rule is to include a vessel that can make at least one feasible trip in Subproblem $_{m}$;

IF vessel $v \in(V-\bar{V}-\underline{V})$ satisfies $\left(t_{v}^{a}+t_{v a s}+t_{v s d}\right) \leq P_{m} \quad \forall a \in A, s \in S, d \in D$,

THEN $\bar{V} \leftarrow \cup\{v\}$

ELSE $\underline{V} \leftarrow \cup\{v\}$;

3.2 SET the cost and time parameters (as listed in Section 4.1) corresponding to $v \in \bar{V}$;

3.3 GENERATE \& SOLVE PBM (using a solver like CPLEX) to find Schedule for Subproblem $_{m}$;

IF $x_{v s d}^{1}=0$ for any $v \in \bar{V}, \bar{V} \leftarrow \bar{V}-\{v\}$ \& REPEAT steps 3.1-3.2; // This guarantees a

better solution by excluding a vessel that is not used in Sub-problem ; $_{\text {; }}$

4. IF $m<M, m \leftarrow m+1 \&$ Repeat steps $1-4$;

\section{References}

Al-Khayyal F, Hwang S (2007) Inventory constrained maritime routing and scheduling for multicommodity liquid bulk, part I: applications and model. Eur J Oper Res 176(1):106-130

Bausch DO, Brown GG, Ronen D (1998) Scheduling short-term marine transport of bulk products. Marit Policy Manag: Flagship J Int Shipping Port Res 25(4):335-348

Bellmore M (1968) A maximum utility solution to a vehicle constrained tanker scheduling problem. Naval Res Logist Quart 15(3):403-411

Bremer WM, Perakis AN (1992) An operational tanker scheduling optimization system: model implementation, results and possible extensions. Marit Policy Manag: Flagship J Int Shipping Port Res 19(3):189-199

Briskin LE (1966) Selecting delivery dates in the tanker scheduling problem. Manag Sci 12(6):B224B235 
Brown GG, Graves GW, Ronen D (1987) Scheduling ocean transportation of crude oil. Manag Sci 33(3):335-346

Chajakis E (2000) Management science for marine petroleum logistics. In: Zanakis SH, Doukidis G, Zopounidis C (eds) Kluwer Academic Publishers, Dordrecht, pp 279-292

Cheng L, Duran MA (2004) Logistics for World-wide crude oil transportation using discrete event simulation and optimal control. Comput Chem Eng 28(6-7):897-911

Christiansen M, Fagerholt K, Ronen D (2004) Ship routing and scheduling: status and perspectives. Transp Sci 38(1):1-18

Christiansen M, Fagerholt K, Nygreen B, Ronen D (2007) Chapter 4 maritime transportation. In: Cynthia B, Gilbert L (eds) Handbooks in operations research and management science. Elsevier, Amsterdam, pp 189-284

Dantzig GB, Fulkerson DR (1954) Minimizing the number of tankers to meet a fixed schedule. Naval Res Logist Quart 1(3):217-222

Deris S, Omatu S, Ohta H, Shaharudin Kutar LC, Abd Samat P (1999) Ship maintenance scheduling by genetic algorithm and constraint-based reasoning. Eur J Oper Res 112(3):489-502

Duffuaa S, Raouf A, Campbell J (1999) Planning and control of maintenance systems: modeling and analysis. Wiley, London

Hennig F, Nygreen B, Furman KC, Song J, Kocis GR (2011) Crude oil tanker routing and scheduling. INFOR: Inform Syst Oper Res Int J 49(2):153-170

Hennig F, Nygreen B, Christiansen M, Fagerholt K, Furman KC, Song J, Kocis GR, Warrick PH (2012) Maritime crude oil transportation-a split pickup and split delivery problem. Eur J Oper Res 218(3):764-774

IBM (2010) IBM ILOG CPLEX Optimizer 12.1. http://www-01.ibm.com/software/commerce/ optimization/cplex-optimizer

Kobayashi K (2010) A linear approximation of the value function of an approximate dynamic programming approach for the ship scheduling problem. Lect Notes Comput Sci 27(1):161-187

Kobayashi K, Kubo M (2010) Optimization of oil tanker schedules by decomposition, column generation, and time-space network techniques. Jpn J Indust Appl Math 27(1):161-173

Kuo T, Chang C (2008) Application of a mathematic programming model for integrated planning and scheduling of petroleum supply networks. Ind Eng Chem Res 47(6):1935-1954

Luo C, Rong G (2009) A Strategy for the integration of production planning and scheduling in refineries under uncertainty. Chin J Chem Eng 17(1):113-127

Mathworks (2004) Matlab 7.0.1.24704 (R14) Service Pack 1. http://www.mathworks.com/products/ matlab/

McKay MD, Hartley HO (1974) Computerized scheduling of seagoing tankers. Naval Res Logist Quart 21(2):255-264

Perakis AN, Bremer WM (1992) An operational tanker scheduling optimization system: background, current practice and model formulation. Marit Policy Manag: Flagship J Int Shipping Port Res 19(3): 177

Rakke JG, Stålhane M, Moe CR, Christiansen M, Andersson H, Fagerholt K, Norstad I (2011) A rolling horizon heuristic for creating a liquefied natural gas annual delivery program. Transp Res Part C: Emerg Technol 19(5):896-911

Rodrigue J, Comtois C, Slack B (2009) The geography of transport systems, Routledge, New York. http:// www.routledge.com/books/details/9780415822541/

Ronen D (1983) Cargo ships routing and scheduling: survey of models and problems. Eur J Oper Res 12(2):119-126

Ronen D (1993) Ship scheduling: the last decade. Eur J Oper Res 71(3):325-333

Sherali HD, Al-Yakoob SM, Hassan MM (1999) Fleet management models and algorithms for an oiltanker routing and scheduling problem. IIE Trans 31(5):395

Siddiqui AW, Ben-Daya M (2009) Reliability centered maintenance. In: Handbook of maintenance management and engineering. Springer, pp 397-415 